\title{
A Neurogenic Component in Muscular Dystrophy
}

\author{
ALAN E. H. EMERY and CHRISTINE GOSDEN \\ University Department of Human Genetics, Western General Hospital, Edinburgh
}

\begin{abstract}
Summary. Evidence for a neurogenic component in mouse and human muscular dystrophy is briefly reviewed. Such evidence comes from certain clinical observations, electrophysiological studies, muscle pathology, nervous system pathology, transplantation experiments in animals, and tissue culture studies. The evidence is at present rather conflicting though the results of recent tissue culture experiments are more convincing. If there is a neurogenic component in dystrophy then the basic defect may have to be sought in the central nervous system rather than in the muscle itself. It is argued, however, that a neurogenic component in dystrophy cannot be simply a defect in the anterior horn cells of the spinal cord since the clinical features and the laboratory and pathological findings are quite different from those in spinal muscular atrophy.
\end{abstract}

The muscular dystrophies are a group of inherited disorders characterized by progressive muscle wasting and weakness and in which the histology of the muscle reveals variation in fibre size, fibre necrosis, and phagocytosis with eventual replacement by proliferating connective tissue and fat. On clinical and genetic grounds a number of different types of muscular dystrophy have been recognized (Table I) but the basic biochemical defect in any of these disorders is unknown.

Until recently the muscular dystrophies were considered to be primary diseases of muscle with the result that biochemists have largely confined their investigations to the study of affected muscle in an attempt to understand the underlying cause. However, the results of some recent studies suggest that there may be a neurogenic component in the pathogenesis of muscular dystrophy. If this proves to be the case then biochemical investigators may wish to turn their attention away from muscle tissue to the study of the nervous system in this group of disorders.

Evidence for a neurogenic component in muscular dystrophy comes from clinical studies and the results of various laboratory investigations. Studies

Received 29 August 1973.
TABLE I

RECOGNIZED TYPES OF MUSCULAR DYSTROPHY
1. $X$-linked
(a) Severe type (Duchenne)
2. Autosomal recessive
(a) Resembling Duchenne
(b) Limb girdle
3. Autosomal dominant
(a) Facioscapulohumeral
(b) Distal
(c) Scapuloperoneal
(d) Ocular
(e) Oculopharyngeal

(b) Benign types (Becker, Mabry, Emery-Dreifuss)

of the pathogenesis of muscular dystrophy have been greatly helped by the discovery of the Bar Harbour 129 strain of mice in which a disorder similar to human muscular dystrophy is inherited as an autosomal recessive trait.

\section{Clinical Studies}

Certain clinical observations have suggested that there may be a neurogenic component in dystrophy. It is known, for example, that a significant proportion of children with Duchenne muscular dystrophy (the commonest form of dystrophy) are mentally 
handicapped (Zellweger and Hanson, 1967) and often have EEG abnormalities (Zellweger and Niedermeyer, 1965). The mental handicap is not a consequence of lack of schooling as a result of physical disability, because the IQ does not change as the disease progresses and mental handicap is not a feature of childhood spinal muscular atrophy in which there is comparable physical disability.

\section{Electrophysiological Studies}

Laboratory evidence for a possible neurogenic component in the pathogenesis of muscular dystrophy first came from the results of electrophysiological studies. In 1965, McComas and Mossawy demonstrated defective motor innervation in the mouse with muscular dystrophy and later showed that in the extensor digitorum brevis of man there was a reduction in the number of motor units in various types of muscular dystrophy (McComas, Sica, and Campbell, 1971). The technique McComas developed for estimating the number of motor units in the extensor digitorum brevis muscle was as follows. Firstly, the average amplitude of single motor unit potentials is determined by stimulating the anterior tibial nerve at the ankle. The amplitude of the muscle potential is then determined by maximal stimulation of the nerve. The number of motor units in the muscle is then obtained by dividing the amplitude of the evoked muscle potential by the average amplitude of single motor unit potentials. McComas and his colleagues (McComas et al, 1971) showed that in patients with muscular dystrophy the size of individual motor unit potentials does not decrease as the disease progresses which would be expected if dystrophy is entirely myopathic in origin and therefore associated with gradual loss of constituent muscle fibres from individual motor units. Instead they found a significant reduction in the number of motor units in the muscle in various types of dystrophy. Harris and Wilson (1971) also demonstrated a reduction in the number of motor units in affected muscle in dystrophic mice.

A reduction in the number of motor units could be explained if there was some defect in the innervation of the muscle centrally. However, it has been argued that, at least in the case of human muscular dystrophy, it is not perhaps justifiable to draw conclusions from studies on a muscle (the extensor digitorum brevis) which is not usually affected until later in the evolution of the disease. For technical reasons it is not possible to study in such a precise manner the number of motor units in an equally accessible muscle which is affected in the disease.
McComas et al (1971) interpreted the results of their investigations as suggesting that muscular dystrophy is associated with 'sick motor neurones': a possible defective production of neurotrophic substance from the anterior horn cells resulting in progressive loss of motor units. Axoplasmic transport of trophic substances necessary for the prevention of denervation changes in muscle is well established (see for example, Hofmann and Thesleff, 1972). Recent observations demonstrate that there is an alteration of axoplasmic flow in mouse dystrophy but this may be a reflection of structural changes in the nerve roots (Bradley and Jaros, 1973).

\section{Muscle Pathology}

Swash (1972) has shown from histological studies that the innervation of muscle spindles is abnormal in myotonic dystrophy. However, the relevance of this to a possible neurogenic component in muscular dystrophy is not clear. If there is defective innervation of skeletal muscle in muscular dystrophy it might be expected that there would be some evidence of group atrophy (Emery, 1971). In a recent study of muscle biopsy specimens from 179 cases of muscular dystrophy Dastur and Razzak (1973) have in fact reported what they consider to be evidence of group atrophy in a proportion of their patients. However, many pathologists who have studied muscle from patients with muscular dystrophy consider that the histology is quite different from that seen in neurogenic atrophy.

\section{Motor End Plate Studies}

Ragab (1971) has investigated the morphology of motor end plates in dystrophic mice, and found that those motor end plates which terminated on apparently healthy fibres were often abnormal. The motor end plate changes in murine dystrophy were characterized by low densities of synaptic vesicles at the nerve endings, the presence of large amounts of filamentous material and the number and complexity of the post synaptic folds was reduced.

Jedrzejczyk et al (1973), in their studies on the motor end plates of chickens homozygous for muscular dystrophy, measured the total amounts of acetycholine and total cholinesterase-like molecules by autoradiographic techniques, and found a significant decrease in the amounts of acetylcholinesterase at the end plates of dystrophic muscles. On the basis of their observations the investigators suggest that a neural factor determining synaptic cholinesterase may be defective in muscular dystrophy. 


\section{Pathology of the Nervous System}

Studies in dystrophic mice have shown a reduction in fibre counts in motor nerves (Harris et al, 1972) and abnormalities in the anterior spinal roots (Bradley and Jenkison, 1973) but similar abnormalities have not been observed in human dystrophy. However, in human muscular dystrophy, at least in the case of children with Duchenne muscular dystrophy with mental retardation, Rosman and Kakulas (1966) have observed abnormalities in the brain at necropsy. These abnormalities included heterotopias and pachygyria.

If muscular dystrophy has a neurogenic origin it is possible that this might be due to a decrease in the number of spinal motor neurones. Counts of the number of spinal motor neurones have shown that in fact there is a significant reduction in mice with spinal muscular atrophy but not apparently in mice with muscular dystrophy (Papapetropoulos and Bradley, 1972) or in human Duchenne muscular dystrophy (J. N. Walton, 1973, personal communication). An investigation of spinal motor neurones at the sub-cellular level has been undertaken by Lin and Hartmann (1971) in rats with drug induced dystrophy. They investigated the base ratios of nuclear and cytoplasmic RNA in normal and dystrophic neurones. Whilst the base ratios from normal anterior horn cell nuclei are indistinguishable from cytoplasmic RNA, in dystrophic neurones the cytoplasmic RNA has a significant decrease in the amount of guanine. The reason for this is not clear but possibly suggests some alteration in the pattern of transcription in spinal motor neurones in dystrophy.

\section{Transplantation Experiments}

The results of transplantation experiments in the mouse have been interpreted as suggesting a neurogenic component in dystrophy. Laird and Timmer (1966), and more recently Hironaka and Miyata (1973), have shown that if muscle tissue was excised from a dystrophic mouse and normal muscle transplanted into the muscle bed, then the latter degenerated, but dystrophic muscle transplanted into normal mice survived well. Salafsky (1971) using a slightly different technique has obtained similar results. If, however, normal muscle is transplanted beneath the kidney capsule of dystrophic mice rather than in the muscle bed, then the muscle does not degenerate (Rolston, 1972) thus suggesting that a humoral factor is unlikely to be responsible for the muscle degeneration. The problems with such transplantation experiments are whether it is the host or donor myoblasts which regenerate, and the interpretation of histological $\stackrel{\Phi}{\Phi}$ changes which may be confounded by changes $\stackrel{\mathbb{Q}}{\vec{D}}$ which are secondary to immune mechanisms, ie, $\stackrel{+}{\rightarrow}$ graft rejection.

\section{Tissue Culture Studies}

The development of techniques for culturing muscle and nerve has opened up a new approach to the study of muscular dystrophy.

In tissue culture, uninucleate myoblasts differentiate to form multinucleate myoblasts with cross striations. It has been observed, however, by Askanas, Shafiq, and Milhorat (1972), Gallup, Strugalska-Cynowska, and Dubowitz (1972), and C. M. Gosden and A. E. H. Emery (1973, unpublished observations) that in both chick and man there is no differentiation into fibre types (that is, no reciprocal pattern of histochemical staining) in tissue culture in the absence of innervation. This contrasts very markedly with the development of fibre types in vivo. It may, however, be significant that there are species differences in the histochemical reactions of muscle cultured in vitro (Gallup et al, 1972) and this fact should stress the need for caution in any extrapolations from animal models to the human condition.

The experimental results of Skeate, Bishop, and Dubowitz (1969) Bishop et al (1971) and Gallup et al (1972) provide evidence that there is no marked difference between normal and dystrophic muscle in culture with regard to growth and differentiation although Bateson, Hindle, and Warren (1972) have reported that in human muscular dystrophy there may be some delay in myotube maturation in culture. In any event the morphology of dystrophic muscle in culture is certainly very similar to normal muscle suggesting that the disorder may not be intrinsically myopathic in origin. Further, if normal mouse muscle is grown in the presence of spinal cord from dystrophic mice, the muscle degenerates but this does not happen if the muscle is grown in the presence of normal spinal cord (Gallup and Dubowitz, 1973). Because of the difficulties of obtaining and growing human dystrophic spinal cord it has not yet been possible to verify these exciting observations in man.

\section{REFERENCES}

Askanas, V., Shafiq, S. A., and Milhorat, A. T. (1972). Histochemistry of cultured aneural chick muscle. Morphological maturation without differentiation of fiber types. Experimental Neurology, 37, 218-230.

Bateson, R. G., Warren, J., and Hindle, D. (1972). Growth patterns in vitro of normal and diseased adult human skeletal muscle. fournal of the Neurological Sciences, 15, 183-191.

Bishop, A., Gallup, B., Skeate, Y., and Dubowitz, V. (1971) Morphological studies on normal and diseased human muscle in culture. Fournal of the Neurological Sciences, 13, 333-350. 
Bradley, W. G. and Jaros, E. (1973). Axoplasmic flow in axonal neuropathies. II. Axoplasmic flow in mice with motor neuron disease and muscular dystrophy. Brain, 96, 247-258.

Bradley, W. G. and Jenkison, M. (1973). Abnormalities of the peripheral nerves in murine muscular dystrophy. Fournal of the Neurological Sciences, 18, 227-247.

Dastur, D. K. and Razzak, Z. A. (1973). Possible neurogenic factor in muscular dystrophy: its similarity to denervation atrophy. fournal of Neurology, Neurosurgery and Psychiatry, 36, 399-410.

Emery, A. E. H. (1971). The nosology of the spinal muscular atrophies. Fournal of Medical Genetics, 8, 481-495.

Gallup, B. and Dubowitz, V. (1973). Failure of 'dystrophic' neurones to support functional regeneration of normal or dystrophic muscle in culture. Nature, 243, 287-289.

Gallup, B., Strugalska-Cynowska, H., and Dubowitz, V. (1972). Histochemical studies on normal and diseased human and chick muscle in tissue culture. Fournal of the Neurological Sciences, 17, 109-125.

Harris, J. B., Wallace, C., and Wing, J. (1972). Myelinated nerve fibre counts in the nerves of normal and dystrophic mouse muscle. fournal of the Neurological Sciences, 15, 245-249.

Harris, J. and Wilson, P. (1971). Denervation in murine dystrophy. Nature, 229, 61-62.

Hironaka, T. and Miyata, Y. (1973). Muscle transplanatation in the aetiological elucidation of murine dystrophy. Nature New Biology, 244, 221-223.

Hofmann, W. W. and Thesleff, S. (1972). Studies on the trophic influence of nerve on skeletal muscle. European fournal of Pharmarcology, 20, 256-260.

Jedrzejczyk, J., Wieckowski, J., Rymaszewska, T., and Barnard, E. A. (1973). Dystrophic chicken muscle: altered synaptic acetylcholinesterase. Science, 180, 406-408.

Laird, J. L. L. and Timmer, R. F. (1966). Transplantation of skeletal muscle into a host with muscular dystrophy. Texas Reports on Biology and Medicine, 24, 169-179.
Lin, J. and Hartmann, H. A. (1971). Nuclear and cytoplasmic RNA in normal and dystrophic neurons. Brain Research, 35, 397-407. McComas, A. J. and Mossawy, S. J. (1965). Electrophysiological investigation of normal and dystrophic muscle in mice. In the Proceedings of Third Symposium of the Muscular Dystrophy Group, Research in Muscular Dystrophy, pp. 317-338. Pitman Medical, London.

McComas, A. J., Sica, R. E. P., and Campbell, M. J. (1971). 'Sick' motoneurones. A unifying concept of muscle disease. Lancet, 1, 321-325.

Papapetropoulos, T. A. and Bradley, W. G. (1972). Spinal motor neurones in murine muscular dystrophy and spinal muscular atrophy. Fournal of Neurology, Neurosurgery and Psychiatry, 35, 60-65.

Ragab, A. H. M. F. (1971). Motor end plate changes in mouse muscular dystrophy. Lancet, 2, 815-816.

Rolston, J. L. L. (1972). Muscle transplanation into mice with muscular dystrophy. Archives of Neurology, 26, 258-264.

Rosman, N. P. and Kakulas, B. A. (1966). Mental deficiency associated with muscular dystrophy. A neuropathological study. Brain, 89, 769-788.

Salafsky, B. (1971). Functional studies of regenerated muscles from normal and dystrophic mice. Nature, 229, 270-272.

Skeate, Y., Bishop, A., and Dubowitz, V. (1969). Differentiation of diseased human muscle in culture. Cell and Tissue Kinetics, 2, 307-310.

Swash, M. (1972). The morphology and innervation of the muscle spindle in dystrophiamyotonica. Brain, 95, 357-368.

Zellweger, H. and Hanson, J. W. (1967). Psychometric studies in muscular dystrophy type IIIa (Duchenne). Developmental Medicine and Child Neurology, 9, 576-581.

Zellweger, H. and Niedermeyer, E. (1965). Central nervous system manifestations in childhood muscular dystrophy (CMD) 1 . Psychometric and electroencephalographic findings. Annales Paediatrici, 205, 25-42. 